\title{
The resurgent character of the Fatou coordinates of a simple parabolic germ
}

\author{
Artem Dudko, David Sauzin
}

\begin{abstract}
Given a holomorphic germ at the origin of $\mathbb{C}$ with a simple parabolic fixed point, the local dynamics is classically described by means of pairs of attracting and repelling Fatou coordinates and the corresponding pairs of horn maps, of crucial importance for ÉcalleVoronin's classification result and the definition of the parabolic renormalization operator. We revisit Écalle's approach to the construction of Fatou coordinates, which relies on BorelLaplace summation, and give an original and self-contained proof of their resurgent character.
\end{abstract}

Keywords: Complex dynamics, Ecalle-Voronin invariants, Resurgent functions.

\section{Resurgence and summability of the formal Fatou coordinate}

Let us give ourselves a simple parabolic germ of holomorphic map at the origin of $\mathbb{C}$, i.e. of the form

$$
F(w)=w+c w^{2}+O\left(w^{3}\right) \in w \mathbb{C}\{w\}
$$

with $c \in \mathbb{C}^{*}$. We rewrite it at $\infty$ by means of the change of coordinate $z=-1 / c w$ :

$$
f(z):=-\frac{1}{c F\left(-\frac{1}{c z}\right)}=z+1+a(z), \quad a(z)=-\rho z^{-1}+O\left(z^{-2}\right) \in z^{-1} \mathbb{C}\left\{z^{-1}\right\}
$$

with some $\rho \in \mathbb{C}$. For every $R>0$ and $\delta \in(0, \pi / 2)$, we introduce the notations

$$
\begin{aligned}
& \mathscr{P}_{R, \delta}^{+}:=\left\{r \mathrm{e}^{\mathrm{i} \theta} \in \mathbb{C} \mid r>R,-\frac{\pi}{2}-\delta<\theta<\frac{\pi}{2}+\delta\right\}, \\
& \mathscr{P}_{R, \delta}^{-}:=\left\{r \mathrm{e}^{\mathrm{i} \theta} \in \mathbb{C} \mid r>R, \frac{\pi}{2}-\delta<\theta<\frac{3 \pi}{2}+\delta\right\} .
\end{aligned}
$$

Definition 1. A pair of Fatou coordinates at $\infty$ is a pair $\left(v^{+}, v^{-}\right)$of univalent maps

$$
v^{+}: \mathscr{P}_{R, \delta}^{+} \rightarrow \mathbb{C}, \quad v^{-}: \mathscr{P}_{R, \delta}^{-} \rightarrow \mathbb{C},
$$

for some $R$ and $\delta$, such that

$$
v^{+} \circ f=v^{+}+1, \quad v^{-} \circ f=v^{-}+1 .
$$

It is well-known that pairs of Fatou coordinates exist and are unique up to the addition of a free pair of constants $\left(c^{+}, c^{-}\right) \in \mathbb{C}^{2}$. We shall see how to recover these facts by means of Borel-Laplace summation.

Notation. Let $b(z):=a(z-1)$ and $b_{1}(z):=b(z)+\rho \log \frac{1+z^{-1} b(z)}{1-z^{-1}}$, so that $b(z) \in z^{-1} \mathbb{C}\left\{z^{-1}\right\}$ and $b_{1}(z) \in z^{-2} \mathbb{C}\left\{z^{-1}\right\}$. We denote by $C_{\mathrm{id}-1}$ and $C_{\mathrm{id}+b}$ the composition operators $\varphi(z) \mapsto \varphi(z-1)$ and $\varphi(z) \mapsto \varphi(z+b(z))$, acting in spaces of functions as well as in the space of formal series $\mathbb{C}\left[\left[z^{-1}\right]\right]$. 
Suppose that $\mathscr{P}=\mathscr{P}_{R, \delta}^{ \pm}$with $R$ large enough so that $b$ is analytic on $\mathscr{P}$ and let Log be any branch of the logarithm in $\mathscr{P}$. Under the change of unknown function $v(z)=z+\rho \log z+\varphi(z)$, the equation $v \circ f=v+1$ is transformed ${ }^{1}$ into

$$
C_{\mathrm{id}-1} \varphi=C_{\mathrm{id}+b} \varphi+b_{1} .
$$

Theorem 1. Equation (4) admits a unique formal solution of the form $\tilde{\varphi}(z)=\sum_{n \geq 0} c_{n} z^{-n-1}$. Its formal Borel transform $\hat{\varphi}:=\mathscr{B} \tilde{\varphi}=\sum_{n>0} c_{n} \frac{\zeta^{n}}{n !}$ is $2 \pi \mathrm{i} \mathbb{Z}$-resurgent, in the sense that it converges for $|\zeta|<2 \pi$ and extends analytically along any path issuing from 0 and staying in $\mathbb{C} \backslash 2 \pi \mathrm{i} \mathbb{Z}$ except for its origin.

Moreover, for such a path $\gamma$ with endpoint $\zeta_{*} \in \mathrm{i} \mathbb{R}$ or for $\gamma=\{0\}$ and $\zeta_{*}=0$, and for $\delta_{0} \in\left(0, \frac{\pi}{2}\right)$, there exist $C_{0}, R_{0}>0$ such that the analytic continuation $\operatorname{cont}_{\gamma} \hat{\varphi}$ of $\hat{\varphi}$ along $\gamma$ (which is a holomorphic germ at $\zeta_{*}$ ) satisfies

$$
\left|\operatorname{cont}_{\gamma} \hat{\varphi}\left(\zeta_{*}+t \mathrm{e}^{\mathrm{i} \theta}\right)\right| \leq C_{0} \mathrm{e}^{R_{0} t} \quad \text { for all } t \geq 0 \text { and } \theta \in I_{\delta_{0}}^{+} \cup I_{\delta_{0}}^{-},
$$

where $I_{\delta_{0}}^{+}:=\left[-\delta_{0}, \delta_{0}\right]$ and $I_{\delta_{0}}^{-}:=\left[\pi-\delta_{0}, \pi+\delta_{0}\right]$.

In the case $\gamma=\{0\}$, inequality (5) says that the formal series $\tilde{\varphi}$ is Borel-Laplace 1-summable in the directions of $I_{\delta_{0}}^{+}$and $I_{\delta_{0}}^{-}$. In fact, Theorem 1 says much more, since it provides a Riemann surface to which $\hat{\varphi}$ extends, with exponential estimates on all its sheets. The rest of this section is devoted to the proof of Theorem 1.

The operator $C_{\mathrm{id}-1}-\mathrm{Id}$ maps $z^{-1} \mathbb{C}\left[\left[z^{-1}\right]\right]$ to $z^{-2} \mathbb{C}\left[\left[z^{-1}\right]\right]$ bijectively, we denote by $E$ the inverse, which decreases the order by one unit and has for Borel counterpart the multiplication operator

$$
\hat{E}: \hat{\psi}(\zeta) \in \zeta \mathbb{C}[[\zeta]] \mapsto \frac{1}{\mathrm{e}^{\zeta}-1} \hat{\psi}(\zeta) \in \mathbb{C}[[\zeta]] .
$$

On the other hand, $B:=C_{\mathrm{id}+b}-\mathrm{Id}=\sum_{k \geq 1} \frac{1}{k !} b^{k}\left(\frac{\mathrm{d}}{\mathrm{d} z}\right)^{k}$ (formally convergent series of operators) increases the order by at least two units, so that the composition $E B$ maps $z^{-k-1} \mathbb{C}\left[\left[z^{-1}\right]\right]$ in $z^{-k-2} \mathbb{C}\left[\left[z^{-1}\right]\right]$ for every $k \geq 0$.

Lemma 1. Let $\tilde{\varphi}_{k}:=(E B)^{k} E b_{1} \in z^{-k-1} \mathbb{C}\left[\left[z^{-1}\right]\right]$ for $k \geq 0$. Then the formally convergent series $\sum_{k \geq 0} \tilde{\varphi}_{k}$ is the unique solution of $(4)$ in $z^{-1} \mathbb{C}\left[\left[z^{-1}\right]\right]$.

Proof. For $\tilde{\varphi} \in z^{-1} \mathbb{C}\left[\left[z^{-1}\right]\right],(4) \Leftrightarrow\left(C_{\mathrm{id}-1}-\mathrm{Id}\right) \tilde{\varphi}=B \tilde{\varphi}+b_{1} \Leftrightarrow \tilde{\varphi}=E B \tilde{\varphi}+E b_{1}$, whence the conclusion follows.

Lemma 2. For each $k \geq 0$ the formal Borel transform $\hat{\varphi}_{k}:=\mathscr{B} \tilde{\varphi}_{k}$ is $2 \pi \mathrm{i} \mathbb{Z}$-resurgent.

Suppose $0<\varepsilon<\pi<\tau, 0<\kappa \leq 1$ and $D$ is a closed disc of radius $\varepsilon$ centred at $2 \pi \mathrm{i} m_{0}$ with $m_{0} \in \mathbb{Z}^{*}$. Then there exist $C_{1}, M, R>0$ such that, for every piecewise $C^{1}$ path with natural parametrisation $\gamma:[0, \ell] \rightarrow \mathbb{C}$ satisfying

$$
\begin{array}{r}
s \in[0, \varepsilon] \Rightarrow|\gamma(s)|=s, \quad s>\varepsilon \Rightarrow|\gamma(s)|>\varepsilon, \\
s \in[0, \ell] \Rightarrow|\gamma(s)|>\kappa s \text { and } \gamma(s) \in \Omega_{\varepsilon, \tau, D}^{+},
\end{array}
$$

\footnotetext{
${ }^{1}$ Notice that, for $|z|$ large enough, $\frac{f(z)}{z}$ is close to 1 , hence $\log f(z)-\log z=\log \frac{f(z)}{z}$ where $\log$ is the principal branch of the logarithm.
} 


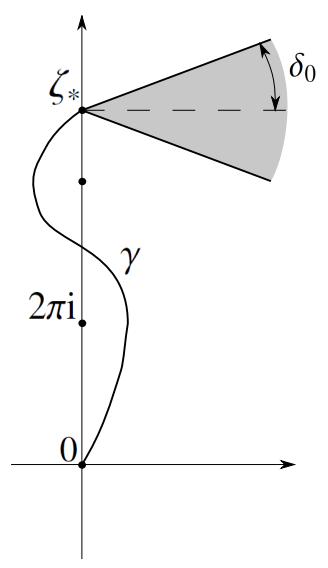

(a) Illustration of Theorem 1 .



(b) Illustration of Lemma 2 .

where $\Omega_{\varepsilon, \tau, D}^{+}:=\left\{\zeta \in \mathbb{C} \mid \operatorname{Re} \zeta>-\tau\right.$, dist $\left.\left(\zeta, 2 \pi \mathrm{i} \mathbb{Z}^{*}\right)>\varepsilon\right\} \backslash\{u \zeta \in \mathbb{C} \mid u \in[1,+\infty), \pm \zeta \in D\}$, one has

$$
\left|\operatorname{cont}_{\gamma} \hat{\varphi}_{k}(\gamma(\ell))\right| \leq C_{1} \frac{(M \ell)^{k}}{k !} \mathrm{e}^{R \ell} \quad \text { for all } k \geq 0 .
$$

Proof. Choose $M_{0}, \lambda>0$ so that $\left|\frac{\zeta}{\mathrm{e}^{\zeta}-1}\right| \leq M_{0} \mathrm{e}^{-\lambda|\zeta|}$ for $\zeta \in \Omega_{\varepsilon, \tau, D}^{+}$. We have $\hat{b}:=\mathscr{B} b, \hat{b}_{1}:=\mathscr{B} b_{1}$ entire functions, with

$$
|\hat{b}(\zeta)| \leq C \mathrm{e}^{\beta|\zeta|} \text { and }\left|\hat{b}_{1}(\zeta)\right| \leq C|\zeta| \mathrm{e}^{\beta|\zeta|} \text { for all } \zeta \in \mathbb{C}
$$

for some $C, \beta>0$. The formal Borel transform maps $b^{k}(z)$ to $\hat{b}^{* k}(\zeta)$, the $k$-th convolution ${ }^{2}$ power of $\hat{b}(\zeta)$, which is also entire and for which we get

$$
\left|\hat{b}^{* k}(\zeta)\right| \leq C^{k} \frac{|\zeta|^{k-1}}{(k-1) !} \mathrm{e}^{\beta|\zeta|} \quad \text { for all } \zeta \in \mathbb{C} .
$$

The Borel counterpart of $C_{\mathrm{id}+b}-\mathrm{Id}$ is $\hat{B}: \hat{\psi} \in \mathbb{C}[[\zeta]] \mapsto \sum_{k \geq 1} \frac{1}{k !} \hat{b}^{* k} *\left((-\zeta)^{k} \hat{\psi}\right) \in \mathbb{C}[[\zeta]]$, which leaves invariant the subspace $\mathbb{C}\{\zeta\}$ and induces on it an integral transform

$$
\hat{B} \hat{\psi}(\zeta)=\int_{0}^{\zeta} K(\xi, \zeta) \hat{\psi}(\xi) \mathrm{d} \xi \quad \text { for } \zeta \text { close to } 0
$$

with kernel function $K(\xi, \zeta)=\sum_{k \geq 1} \frac{(-\xi)^{k}}{k !} \hat{b}^{* k}(\zeta-\xi)$. The point is that this kernel is holomorphic in $\mathbb{C} \times \mathbb{C}$, with estimates following from $(9)$ :

$$
|K(\xi, \zeta)| \leq C|\xi| \mathrm{e}^{\mu|\xi|+\left(\frac{C}{\mu}+\beta\right)|\zeta-\xi|} \text { for all }(\xi, \zeta) \in \mathbb{C} \times \mathbb{C},
$$

with any $\mu>0$. Therefore, whenever a germ $\hat{\psi} \in \mathbb{C}\{\zeta\}$ admits analytic continuation along a naturally parametrised path $\gamma:[0, \ell] \rightarrow \mathbb{C}$, this is also true for $\hat{B} \hat{\psi}$, with $\operatorname{cont}_{\gamma} \hat{B} \hat{\psi}(\gamma(s))=$ $\int_{0}^{s} K(\gamma(\sigma), \gamma(s)) \operatorname{cont}_{\gamma} \hat{\psi}(\gamma(\sigma)) \gamma^{\prime}(\sigma) \mathrm{d} \sigma$ for all $s \in[0, \ell]$. Hence $2 \pi \mathrm{i} \mathbb{Z}$-resurgence is preserved by the composition $\hat{E} \hat{B}$ and we can get estimates.

\footnotetext{
${ }^{2}$ The formal Borel transform $\mathscr{B}$ maps the Cauchy product of formal series to the convolution product defined by $\hat{\phi} * \hat{\psi}(\zeta)=\int_{0}^{\zeta} \hat{\phi}(\zeta-\xi) \hat{\psi}(\xi) \mathrm{d} \xi$, with termwise integration for formal series $\hat{\phi}, \hat{\psi} \in \mathbb{C}[[\zeta]]$, and with the obvious analytical meaning when $\hat{\phi}, \hat{\psi} \in \mathbb{C}\{\zeta\}$ (then taking $\zeta$ close enough to 0 ).
} 
We start with $\hat{\varphi}_{0}=\hat{E} \hat{b}_{1}=\frac{\hat{b}_{1}(\zeta)}{\mathrm{e}^{\zeta}-1}$, which is meromorphic and clearly $2 \pi \mathrm{i} \mathbb{Z}$-resurgent, and satisfies (8) with $C_{1}:=M_{0} C$ and with any $R \geq \beta$. We choose $\mu:=\lambda \kappa, R:=\frac{C}{\mu}+\beta$ and $M:=\frac{M_{0} C}{\kappa}$. We obtain the desired result for $\hat{\varphi}_{k}=(\hat{E} \hat{B})^{k} \hat{\varphi}_{0}$ by induction, observing that if a path $\gamma$ satisfies (6)-(7) and a $2 \pi$ iZZ-resurgent function $\hat{\psi}$ satisfies $\left|\operatorname{cont}_{\gamma} \hat{\psi}(\gamma(s))\right| \leqslant \mathrm{e}^{R s} \Psi(s)$ for all $s \in[0, \ell]$ then, for all $0 \leqslant \sigma \leqslant s \leqslant \ell,|K(\gamma(\sigma), \gamma(s))| \leqslant C \sigma \mathrm{e}^{\mu \sigma+R(s-\sigma)}$ and $\left|\frac{1}{\mathrm{e}^{\gamma(s)}-1}\right| \leq \frac{M_{0}}{\kappa s} \mathrm{e}^{-\mu s}$, whence

$$
\left|\operatorname{cont}_{\gamma} \hat{E} \hat{B} \hat{\psi}(\gamma(s))\right| \leqslant \frac{C M_{0}}{\kappa} \mathrm{e}^{R s} \int_{0}^{s} \frac{\sigma}{s} \Psi(\sigma) \mathrm{e}^{-\mu(s-\sigma)} \mathrm{d} \sigma \leqslant M \mathrm{e}^{R s} \int_{0}^{s} \Psi(\sigma) \mathrm{d} \sigma \quad \text { for all } s \in[0, \ell] .
$$

We can deduce that the series of holomorphic functions $\sum_{k \geq 0} \hat{\varphi}_{k}$ converges normally in any compact subset of the disc $\{|\zeta|<2 \pi\}$ (using paths $\gamma$ of the form $[0, \zeta]$ ) and that its sum, which is $\hat{\varphi}$, extends analytically along any naturally parametrised path $\gamma$ which starts as the line segment $[0,1]$ and then stays in $\mathbb{C} \backslash 2 \pi \mathrm{i} \mathbb{Z}$ : indeed, taking $\varepsilon, \kappa$ small enough and $\tau, m_{0}$ large enough, we see that Lemma 2 applies to $\gamma$ and the neighbouring paths, so that (8) yields the normal convergence of $\sum_{k \geq 0} \operatorname{cont}_{\gamma} \hat{\varphi}_{k}(\gamma(t)+\zeta)=\operatorname{cont}_{\gamma} \hat{\varphi}(\gamma(t)+\zeta)$ for all $t$ and $\zeta$ with $|\zeta|$ small enough. Therefore $\hat{\varphi}$ is $2 \pi \mathrm{i} \mathbb{Z}$-resurgent.

We also get the part of (5) relative to $I_{\delta_{0}}^{+}$: given $\delta_{0} \in(0, \pi / 2)$ and $\gamma$ with endpoint $\zeta_{*} \in \mathrm{i} \mathbb{R}$, we first replace an initial portion of $\gamma$ with a line segment of length 1 (unless $\gamma$ stays in the unit disc, in which case the modification of the arguments which follow is trivial) and switch to its natural parametrisation $\gamma:[0, \ell] \rightarrow \mathbb{C}$. We then choose

$$
\begin{aligned}
& \varepsilon<\min \left\{1, \min _{[1, \ell]}|\gamma|, \operatorname{dist}\left(\gamma([0, \ell]), 2 \pi \mathrm{i} \mathbb{Z}^{*}\right), \operatorname{dist}\left(\zeta_{*}, 2 \pi \mathrm{i} \mathbb{Z}\right) \cos \delta_{0}\right\}, \\
& \kappa<\min \left\{\min _{[0, \ell]} \frac{|\gamma(s)|}{s}, \min _{t \geq 0} \frac{\mid \zeta_{*}+t \mathrm{e}^{ \pm \mathrm{i} \delta_{0} \mid}}{\ell+t}\right\},
\end{aligned}
$$

$\tau>-\min \operatorname{Re} \gamma$ and $m_{0}>\frac{1}{2 \pi}(\varepsilon+\max |\operatorname{Im} \gamma|)$, so that, for each $t \geq 0$ and $\theta \in I_{\delta_{0}}^{+}$, Lemma 2 applies to the concatenation $\Gamma:=\gamma+\left[\zeta_{*}, \zeta_{*}+t \mathrm{e}^{\mathrm{i} \theta}\right]$; since $\Gamma$ has length $\ell+t$, (8) yields $\left|\operatorname{cont}_{\gamma} \hat{\varphi}\left(\zeta_{*}+t \mathrm{e}^{\mathrm{i} \theta}\right)\right|=\left|\operatorname{cont}_{\Gamma} \hat{\varphi}(\Gamma(\ell+t))\right| \leq C_{1} \mathrm{e}^{(M+R)(\ell+t)}$.

The part of $(5)$ relative to $I_{\delta_{0}}^{-}$follows from the fact that $\hat{\varphi}^{-}(\zeta):=\hat{\varphi}(-\zeta)$ satisfies all the properties we just obtained for $\hat{\varphi}(\zeta)$, since it is the formal Borel transform of $\tilde{\varphi}^{-}(z):=-\tilde{\varphi}(-z)$ which solves the equation $C_{\mathrm{id}-1} \tilde{\varphi}^{-}=C_{\mathrm{id}+b^{-}} \tilde{\varphi}^{-}+b_{*}^{-}$associated with the simple parabolic germ $f^{-}(z):=-f^{-1}(-z)=z+1+b^{-}(z+1)$.

The proof of Theorem 1 is now complete.

Remark 1. The name "resurgent", whose definition in a particular case is given in the statement of Theorem 1, was chosen by Écalle because of the explicit connection he obtained between the germ $\hat{\varphi}$ at 0 and the singularities of its analytic continuation at any point of $2 \pi \mathrm{i} \mathbb{Z}$ - see [DS13]. Theorem 1 is stated in [Eca81], with a detailed proof only for the case $\rho=0$. The above proof is original and simpler.

Remark 2. For a path $\gamma$ ending at a point $\zeta$, we get the explicit formula

$$
\operatorname{cont}_{\gamma} \hat{\varphi}(\zeta)=\frac{1}{\mathrm{e}^{\zeta}-1}\left(\hat{b}_{1}(\zeta)+\sum_{k \geqslant 1} \int_{\Delta_{\gamma, k}} \hat{b}_{1}\left(\xi_{1}\right) \frac{K\left(\xi_{1}, \xi_{2}\right) \cdots K\left(\xi_{k-1}, \xi_{k}\right) K\left(\xi_{k}, \zeta\right)}{\left(\mathrm{e}^{\xi_{1}}-1\right) \cdots\left(\mathrm{e}^{\xi_{k-1}}-1\right)\left(\mathrm{e}^{\xi_{k}}-1\right)} \mathrm{d} \xi_{1} \wedge \cdots \wedge \mathrm{d} \xi_{k}\right)
$$

with the notation $\Delta_{\gamma, k}:=\left\{\left(\gamma\left(s_{1}\right), \ldots, \gamma\left(s_{k}\right)\right) \mid s_{1} \leqslant \cdots \leqslant s_{k}\right\}$ for each positive integer $k$. 


\section{Fatou coordinates and lifted horn maps}

For $\gamma=\{0\}$ and any $\delta_{0} \in(0, \pi / 2)$, Theorem 1 provides $R_{0}=R_{0}\left(\delta_{0}\right)$ and we may apply the Laplace transform:

$$
\mathcal{L}^{\theta} \hat{\varphi}(z):=\int_{0}^{\mathrm{e}^{\mathrm{i} \theta} \infty} \mathrm{e}^{-z \zeta} \hat{\varphi}(\zeta) \mathrm{d} \zeta, \quad \theta \in I_{\delta_{0}}^{+} \cup I_{\delta_{0}}^{-},
$$

defining $\mathcal{L}^{\theta} \hat{\varphi}$ holomorphic in $\Pi_{R_{0}\left(\delta_{0}\right)}^{\theta}:=\left\{z \in \mathbb{C} \mid \operatorname{Re}\left(z \mathrm{e}^{\mathrm{i} \theta}\right)>R_{0}\left(\delta_{0}\right)\right\}$. The functions $\mathcal{L}^{\theta} \hat{\varphi}$ with $\theta \in I_{\delta_{0}}^{+}$are extension of each other, similarly for $I_{\delta_{0}}^{-}$, so we get two Borel-Laplace sums

$$
\mathcal{L}^{+} \hat{\varphi} \text { in } U^{+}:=\bigcup_{\delta_{0} \in\left(0, \frac{\pi}{2}\right)} \bigcup_{\theta \in I_{\delta_{0}}^{+}} \Pi_{R_{0}\left(\delta_{0}\right)}^{\theta}, \quad \mathcal{L}^{-} \hat{\varphi} \text { in } U^{-}:=\bigcup_{\delta_{0} \in\left(0, \frac{\pi}{2}\right)} \bigcup_{\theta \in I_{\delta_{0}}^{-}} \Pi_{R_{0}\left(\delta_{0}\right)}^{\theta}
$$

Since $U^{ \pm} \subset \mathbb{C} \backslash \mathbb{R}^{\mp}$, we choose branches of the logarithm as follows:

$$
\log ^{+} z=\int_{1}^{z} \frac{\mathrm{d} u}{u} \text { for } z \in \mathbb{C} \backslash \mathbb{R}^{-}, \quad \log ^{-} z=\mathrm{i} \pi+\int_{-1}^{z} \frac{\mathrm{d} u}{u} \text { for } z \in \mathbb{C} \backslash \mathbb{R}^{+} .
$$

Theorem 2. The formulas

$$
v_{*}^{ \pm}(z):=z+\rho \log ^{ \pm} z+\mathcal{L}^{ \pm} \hat{\varphi}(z), \quad z \in U^{ \pm},
$$

define a pair $\left(v_{*}^{+}, v_{*}^{-}\right)$of Fatou coordinates at $\infty$ for the simple parabolic germ $f$. For any $0<\delta^{\prime}<\delta<\pi / 2$, provided that $R$ is large enough, the function $\mathcal{L}^{ \pm} \hat{\varphi}$ has uniform 1-Gevrey asymptotic expansion $\tilde{\varphi}$ in $\mathscr{P}_{R, \delta}^{ \pm}$and the function $v_{*}^{ \pm}$is univalent in $\mathscr{P}_{R, \delta}^{ \pm}$with its image containing a set of the form $\mathscr{P}_{R^{\prime}, \delta^{\prime}}^{ \pm}$.

Any other pair of Fatou coordinates is of the form $\left(v_{*}^{+}+c^{+}, v_{*}^{-}+c^{-}\right)$with arbitrary complex constants $c^{+}, c^{-}$.

(For the definition and properties of 1-Gevrey asymptotic expansion, see [Mal95] or [Ram93].)

Proof. Since $b_{1}$ is convergent, it coincides with $\mathcal{L}^{+} \hat{b}_{1}$ on $U^{+}$, similarly $b_{\mid U^{+}}=\mathcal{L}^{+} \hat{b}$. We have $\mathrm{e}^{\zeta} \hat{\varphi}=\hat{\varphi}+\hat{B} \hat{\varphi}+\hat{b}_{1}$ and $\mathcal{L}^{+}\left(\mathrm{e}^{\zeta} \hat{\varphi}\right)(z)=\left(\mathcal{L}^{+} \hat{\varphi}\right)(z-1)$. The Laplace transform maps the convolution product to the product of functions and the operator of multiplication by $-\zeta$ to the differentiation $\frac{\mathrm{d}}{\mathrm{d} z}$, hence its action on each term of $\hat{\varphi}+\hat{B} \hat{\varphi}=\hat{\varphi}+\sum_{k \geq 1} \frac{1}{k !} \hat{b}^{* k} *$ $\left((-\zeta)^{k} \hat{\varphi}\right)$ is clear and (9) allows us to exchange $\mathcal{L}^{+}$and $\sum$ :

$$
\mathcal{L}^{+}(\hat{\varphi}+\hat{B} \hat{\varphi})=\sum_{k \geq 0} \frac{1}{k !} b^{k}\left(\frac{\mathrm{d}}{\mathrm{d} z}\right)^{k} \mathcal{L}^{+} \hat{\varphi}=\mathcal{L}^{+} \hat{\varphi} \circ(\mathrm{id}+b) .
$$

It follows that $\mathcal{L}^{+} \hat{\varphi}$ is a solution of (4), and hence that $v_{*}^{+}$

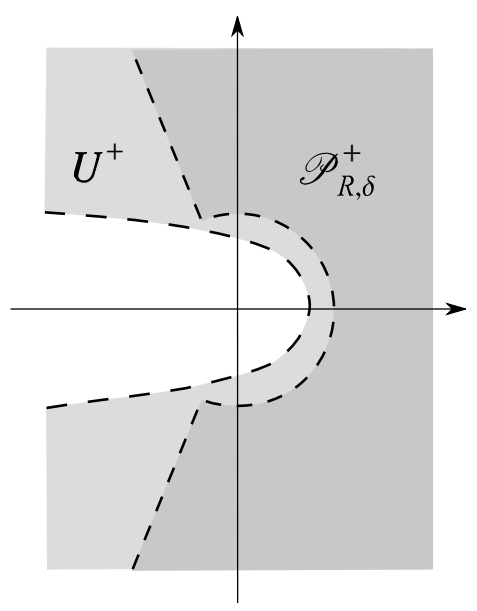

(c) Illustration of Theorem 2. satisfies (3).

Observe that $\mathscr{P}_{R, \delta}^{+} \subset U^{+}$as soon as there exists $\delta_{0}$ such that $\delta<\delta_{0}$ and $R \geq R_{0}\left(\delta_{0}\right) / \sin \left(\delta_{0}-\right.$ $\delta)$. The 1-Gevrey asymptotic property is standard in Borel-Laplace summation. It implies $\mathcal{L}^{+} \hat{\varphi}=O\left(z^{-1}\right), \frac{\mathrm{d}}{\mathrm{d} z}\left(\mathcal{L}^{+} \hat{\varphi}\right)=O\left(z^{-2}\right)$, and also the univalence of $v_{*}^{+}$in $\mathscr{P}_{R, \delta}^{+}$for $R$ large enough and the statement on its image, because $\left|\frac{v_{*}^{+}(z)}{z}\right| \rightarrow 1$ and $\frac{v_{*}^{+}(z)}{\left|v_{*}^{+}(z)\right|} \sim \frac{z}{|z|}$. 
For any univalent solution $v^{+}$of $(3)$ on $\mathscr{P}_{R, \delta}^{+}$, the univalent function $v^{+} \circ\left(v_{*}^{+}\right)^{-1}: \mathscr{P}_{R^{\prime}, \delta^{\prime}}^{+} \rightarrow$ $\mathbb{C}$ conjugates id +1 with itself, hence is of the form id $+P$ with a 1-periodic $P$ and extends univalently to $\mathbb{C}$, hence $P$ is constant. For $v_{*}^{-}$the proof is similar.

Remark 3. The first use of Borel-Laplace summation for obtaining Fatou coordinates is in [Eca81, §9c]. The asymptotic property without the Gevrey qualification can be found in earlier works by G. Birkhoff, G. Szekeres, T. Kimura and J. Écalle - see [Lor05]; see [LY12] for a recent independent proof and an application to numerical computations.

For any pair of Fatou coordinates $\left(v^{+}, v^{-}\right)$, we can take $\delta^{\prime}<\delta$ and large $R, R^{\prime}$ so that $\mathscr{P}_{R, \delta}^{+} \cap\left(v^{-}\right)^{-1}\left(\mathscr{P}_{R^{\prime}, \delta^{\prime}}^{-}\right)$has upper and lower connected components $\mathscr{Q}^{\text {up }}$ and $\mathscr{Q}^{\text {low }}$. The lifted horn maps are $h^{\text {up }}:=v^{+} \circ\left(v^{-}\right)_{\mid \mathscr{Q}^{\text {up }}}^{-1}$, which conjugates id +1 with itself, hence is of the form id $+P^{\text {up }}$ with a 1-periodic $P^{\text {up }}$ and extends to an upper half-plane, and $h^{\text {low }}:=v^{+} \circ\left(v^{-}\right)_{\mid \mathscr{Q}^{\text {low }}}^{-1}$ with similar properties in a lower half-plane. For the normalized pair $\left(v_{*}^{+}, v_{*}^{-}\right)$we get $h_{*}^{\text {up } / \text { low }}(Z)=$ $Z+\rho\left(\log ^{+} Z-\log ^{-} Z\right)+o(1)$, where $\log ^{+}-\log ^{-} \equiv 0$ in $\mathscr{Q}^{\text {up }}$ and $\equiv-2 \pi$ i in $\mathscr{Q}^{\text {low }}$, which puts a constraint on the form of the Fourier expansion of $h_{*}^{\text {up/low }}-$ id:

$$
h_{*}^{\text {up }}(Z)-Z=\sum_{m \geq 1} A_{m} \mathrm{e}^{2 \pi \mathrm{i} m Z}, \quad h_{*}^{\text {low }}(Z)-Z=-2 \pi \mathrm{i} \rho+\sum_{m \geq 1} A_{-m} \mathrm{e}^{-2 \pi \mathrm{i} m Z} .
$$

Definition 2. The coefficients $A_{m}, m \in \mathbb{Z}^{*}$, are called the Écalle-Voronin invariants of the simple parabolic germ $f$.

This name is motivated by Écalle-Voronin's classification result ([Eca81], [Vor81], [Mal82]): (i) two simple parabolic germs $f, g$ are analytically conjugate if and only if there exists $c \in \mathbb{C}$ such that, for all $m, A_{m}(g)=\mathrm{e}^{2 \pi i m c} A_{m}(f)$ (direct consequence of the results of this section); (ii) any pair of Fourier series of the form $\left(\sum_{m \geq 1} A_{m} \mathrm{e}^{2 \pi \mathrm{i} m Z}, A_{0}+\sum_{m \geq 1} A_{-m} \mathrm{e}^{-2 \pi \mathrm{i} m Z}\right)$, where the first (resp. second) one is holomorphic in an upper (resp. lower) half-plane, can be obtained as $\left(h_{*}^{\text {up }}-\right.$ id, $h_{*}^{\text {low }}-$ id) for a simple parabolic germ $f$ (this part of the result is more difficult).

By definition, the horn maps $H_{*}^{\text {up/low }}(w)$ are the lifted horn maps $h_{*}^{\text {up/low }}$ represented in the coordinate $w=\mathrm{e}^{2 \pi \mathrm{i} Z}$, i.e. $H_{*}^{\mathrm{up} / \text { low }}(w):=\exp \left(2 \pi \mathrm{i} h_{*}^{\mathrm{up} / \mathrm{low}}(z)\right)$, so that one gets a germ at 0 and a germ at $\infty$ on the Riemann sphere:

$H_{*}^{\mathrm{up}}(w)=w+2 \pi \mathrm{i} A_{1} w^{2}+O\left(w^{3}\right) \in w \mathbb{C}\{w\}, \quad H_{*}^{\text {low }}(w)=\mathrm{e}^{4 \pi^{2} \rho}\left(w+2 \pi \mathrm{i} A_{-1}+O\left(w^{-1}\right)\right) \in w \mathbb{C}\left\{w^{-1}\right\}$.

Some authors also refer to them as the Écalle-Voronin invariants. The parabolic renormalization operator $\mathscr{R}$, of great importance in recent developments of complex dynamics, is defined by $\mathscr{R}: F \mapsto H_{*}^{\text {up }}$ (recall that $F(w)=w+c w^{2}+O\left(w^{3}\right) \in w \mathbb{C}\{w\}$ was assumed to be a simple parabolic germ at 0 , i.e. $c \neq 0$, and notice that $\mathscr{R} F$ is itself a parabolic germ at 0 , but not necessarily simple: this requires $A_{1} \neq 0$ ).

In this article, we have only exploited the information on the principal branch of $\hat{\varphi}$ given by Theorem 1. The reader is referred to [DS13] for an investigation of the singularities of all the branches of $\hat{\varphi}$ and their relation to the Écalle-Voronin invariants, which shows how the horn maps are encoded in the Borel plane. 
Acknowledgements. The authors are grateful to F. Fauvet and M. Yampolsky for fruitful discussions and encouragements. The first author expresses thanks to the Centro di Ricerca Matematica Ennio De Giorgi and Fibonacci Laboratory for hospitality. The second author acknowledges the support of the French National Research Agency under the reference ANR-12-BS01-0017.

\section{References}

[DS13] A. Dudko, D. Sauzin. On the resurgent approach to Écalle-Voronin's invariants. Preprint oai:hal.archives-ouvertes.fr:hal-00849401, 2013.

[Eca81] J. Écalle. Les fonctions résurgentes, Vol. 1 \& 2. Publ. Math. d'Orsay 81-05 \& 81-06, 1981.

[Lor05] F. Loray. Pseudo-groupe d'une singularité de feuilletage holomorphe en dimension deux. Preprint oai:hal.archives-ouvertes.fr:hal-00016434, 2005.

[LY12] O. Lanford III, M. Yampolsky. The fixed point of the parabolic renormalization operator. Preprint arXiv:1108.2801, 2012.

[Mal82] B. Malgrange. Travaux d'Écalle et de Martinet-Ramis sur les systèmes dynamiques. Séminaire Bourbaki, 1981/82, Exposé 582. Astérisque 92-93 (1982), 59-73.

[Mal95] B. Malgrange. Sommation des séries divergentes. Exp. Math. 13 (1995), 163-222.

[Ram93] J.-P. Ramis. Séries divergentes et théories asymptotiques. Bull. Soc. Math. France (suppl.), Panoramas et Synthèses, v. 121. Paris: Société Mathématique de France. 74 pp., 1993.

[Vor81] S. Voronin, Analytic classification of germs of conformal mappings $(\mathbb{C}, 0) \rightarrow(\mathbb{C}, 0)$. (Russian) Funktsional. Anal. i Prilozhen 15 (1981), no. 1, 1-17, 96. (English translation: Functional Anal. Appl. 15 (1981), no. 1, 1-13.)

Artem Dudko

Institute for Mathematical Sciences

University of Stony Brook, NY, USA

David Sauzin

CNRS UMI 3483 - Laboratoire Fibonacci,

Centro di Ricerca Matematica Ennio De Giorgi,

Scuola Normale Superiore di Pisa, Italy 